\title{
Laminar flow through fractal porous materials: the fractional-order transport equation
}

\author{
Gianluca Alaimo, Massimiliano Zingales* \\ Dipartimento di Ingegneria Civile, Ambientale, Aerospaziale e dei Materiali (DICAM), Viale delle Scienze ed. 8, 90128 Palermo, Italy \\ Laboratorio di Biomeccanica e Nanomeccanica per le scienze mediche, Viale delle Scienze ed. 18, 90128 Palermo, Italy
}

\section{A R T I C L E I N F O}

\section{Article history:}

Received 22 May 2014

Received in revised form 8 October 2014

Accepted 9 October 2014

Available online 24 October 2014

\section{Keywords:}

Transport equations

Fractional calculus

Fractals

\begin{abstract}
A B S T R A C T
The anomalous transport of a viscous fluid across a porous media with power-law scaling of the geometrical features of the pores is dealt with in the paper. It has been shown that, assuming a linear force-flux relation for the motion in a porous solid, then a generalized version of the Hagen-Poiseuille equation has been obtained with the aid of RiemannLiouville fractional derivative. The order of the derivative is related to the scaling property of the considered media yielding an appropriate mechanical picture for the use of generalized fractional-order relations, as recently used in scientific literature.
\end{abstract}

(c) 2014 Elsevier B.V. All rights reserved.

\section{Introduction}

Molecular diffusion is an efficient mechanism of passive mass transport that is frequently encountered in several field of physics, biology and engineering sciences. Indeed, as an example, the transport of thermal energy by conduction is due to the diffusion of phonon carrier across the material. In a similar fashion the passive transport of chemical species across biological membranes is ruled by diffusion mechanism as well as the transport of fluids across porous media as frequently encountered in geotechnical engineering.

The usual approach to mass diffusion is conducted by means of linear force-flux relations that relates the mass transfer rate and the flux to the gradient of a driving force that may be gradient of pressure, concentration or temperature respectively in fluid, chemical or heat transport phenomena. These approaches became very popular in last century's research with continuum mechanics to yield multi-field theories.

Mathematical model of coupled multi-field problems encountered in recent advanced applications of physical science and engineering requires, however, the introduction of more complete operators to account for scale as well as of non-uniform geometrical features of the considered domain. In this regard the use of fractional-order differential calculus proved to be one of the most powerful method to handle physical complexity [1,2]. Indeed the generalization of integro-differential operators of classical differentiation proved to be useful in several fields of physical sciences and engineering [3-5].

The main feature of these approaches relies in the "naive" replacement of the differential operators of the classical continuum field theories with their real-order counterpart. However the physical and geometrical aspects beyond this replacement remain hidden and only in very recent contributions the authors had provided some physical insights beyond fractional operators in mechanics, material sciences and heat transport [6-14].

\footnotetext{
* Corresponding author at: Dipartimento di Ingegneria Civile, Ambientale, Aerospaziale e dei Materiali (DICAM), Viale delle Scienze ed. 8, 90128 Palermo, Italy.

E-mail address: massimiliano.zingales@unipa.it (M. Zingales).
} 
The recent achievements in the physics of fractional-order operators had also used by the authors to introduce some connections among the exponent of power-law decay of time dependent phenomena as stress relaxation in biological and polymeric materials [15] and the scaling exponent of non-euclidean geometry. Indeed the introduction of such a relation is a crucial aspect in several fields of applied physics and engineering as the biomedical and biomechanics setting where the chance to provide some mechanical parameters from non invasive diagnosis represents an important step.

The anomalous diffusion has been recently discussed in terms of the 1D flux of a Newtonian fluid across a functionally graded porous media [6]. In more details, the governing equations of the flux across the media, represented by the state equation of the saturated media, the continuity condition as well as the Darcy transport equations have been used to describe the pressure field. The variation of the physical parameters involved the compressibility of the saturated media and the porosity. It has been observed that, as the functional grading belongs to power-law functional class, then the observed flux across a specified control section of the media is related to the pressure gradient by a Riemann-Liouville fractional-order derivative. The real-order exponent of the fractional derivative is related to the grading of the physical properties of the media, that is to the spatial scaling of the compressibility and the porosity of the saturated medium.

The results obtained in previous paper showed that there is a specific relation among the fractional differentiation order and the scaling exponent of the physical properties of the medium in which the flux is occurring. This consideration, however, does not answer a fundamental question: "Why power-law spatial scaling of the physical properties of the media yields transport equations ruled by fractional-order operators related to the scaling exponent?".

The existence of a bridge among fractal geometry and fractional order physics is a well-known topic of physics and mathematical physics and since the mid of the nineties of the last century, several researches aimed to provide a connection among fractal geometry and physical operators as fractional derivatives. Among them, important contributions have been provided in papers $[16,17]$ in which the physical representation of the fractional order integral has been related to the presence of reachable states of physical systems that curdles in non-dense region of the real axis. In other papers a local version of fractional-order calculus has been introduced to handle functions defined on non-dense sets in several fields of physics and engineering [18-20].

The arguments provided in previous papers, despite their generality, do not deal with the introduction of a specific physical system that is not ruled in a mechanical perspective by fractional-order operators.

In this paper the authors aim to introduce a mechanical model of fluid mass transport across a fractal porous media that involves anomalous scaling of the memory function. The exponent of the scaling is related to the fractal dimension of the pores of the set and, in this regard, we may confirm the quotation in [16,21]: "Some physical system that can be described by equations in fractional derivatives must contain channels belonging to some branching fractal structure".

In the paper it will be assumed that the flow occurs in a self-similar porous volume with prescribed fractal dimension $d$ and it will be shown that in presence of flow of a viscous fluid in the Poiseuille laminar regime, the flux of the initially contained fluid is related to the pressure gradient fluctuations by means of a Riemann-Liouville fractional-order derivative. The differentiation-order is related to the fractal dimension of the porous volume yielding a direct connections among fractals and fractional differential operators.

This result does not sound as a new discovery in physics as well as in the context of applied analysis but, in this paper, it has been obtained in the context of classical mechanics. This is the novelty of the present study, showing that also without resorting to methodologies of the statistical physics [22,23], phenomena occurring on fractal sets yield time variation of the state variables in terms of power-laws, with the exponent related to the dimension of the underlying fractal set.

This paper is organized reporting in the next sections some remarks of fractal geometry to be acquainted with the fundamental definitions. The transport across fractal media will be discussed in Section 3 with some numerical examples (Sections 3.1.1 and 3.1.2), while the relation with fractional-order operators will be introduced in Section 3.2. Some conclusions will be withdrawn in Section 4.

\section{Remarks on fractal geometry}

Fractal objects, dubbed $F$, enjoy the self-similarity property so that, $F \subseteq \mathbb{R}^{m}$ is self-similar if it may be defined as the union of self-similar copies of itself. Such an example, the middle third Cantor set $F$ in the interval $\left[0, L_{0}\right]$ is the union of two fundamental similarities $S^{(i)}:\left[0, L_{0}\right] \rightarrow\left[0, L_{0}\right]$ with $i=1,2$ defined as:

$$
S^{(1)}(x)=L_{0}\left(\frac{x}{3}\right) ; \quad S^{(2)}(x)=L_{0}\left(\frac{2}{3}+\frac{x}{3}\right)
$$

that means the exclusion of the middle third from each previous level as shown in Fig. 1.

The zero-th level, namely $E^{(0)}=\left[0, L_{0}\right]$ is dubbed parent object or initiator, whereas the first level of the fractal $E^{(1)}=\left[0, L_{0} / 3\right] \cup\left[2 / 3 L_{0}, L_{0}\right]$ is known as generator, the intermediate level $E^{(k)}$ is the $k^{\text {th }}$ pre-fractal of $F$ and $E^{(k)}$ with $k \rightarrow \infty$ is the fractal set $F$, known as Cantor dust.

It may be observed that, the Euclidean topological measure of the length of the $k$ th pre-fractal set $E^{(k)}$ is $\left(2^{k} / 3^{k}\right) L_{0}$ so that the fractal set possesses an Euclidean, topological length approaching zero at the limit as $k \rightarrow \infty$. On the other hand since the Cantor dust is the sum of $2^{k}$ segments (of infinitesimal length $L_{0} / 3^{k}$ as $k \rightarrow \infty$ ) then the classical topological measure, simply 


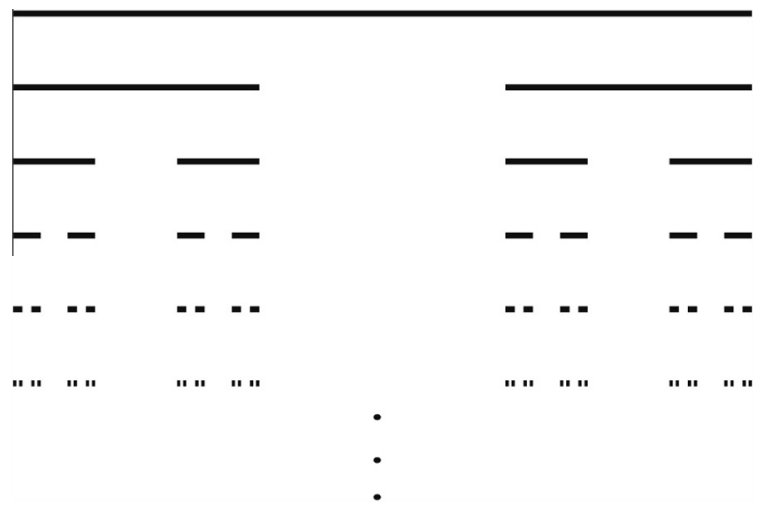

Fig. 1. Cantor set.

evaluated as a classical Euclidean object fails since the fractal dust is not a set of isolated points (topological Euclidean dimension 0) nor a set of lines (topological Euclidean dimension 1).

The fundamental intuition of [24] relies in the observation that by changing the observation scale $E^{(k-1)} \rightarrow E^{(k)}$, so that by introducing new details in the description of the set, an invariant quantity appear. That is the number $N$ of segments at the $N$ th level multiplied by the corresponding topological length, i.e. $\left(r_{N} L_{0}\right)$, in power $d_{M}$, remains constant at any resolution scale, namely

$$
N\left(r_{N} L_{0}\right)^{d_{M}}=L_{0}^{d_{M}} \Rightarrow d_{M}=-\frac{\ln (N)}{\ln \left(r_{N}\right)} ; \quad \forall N
$$

where $d_{M}$ is the Mandelbrot dimension definition of the fractal set. For the Cantor dust at the $k$ th level (pre-fractal $\left.E^{(k)}\right) N=2^{k}$ and $r_{N}=1 / 3^{k}$ so that from Eq. (2) the Mandelbrot dimension reads $d_{M}=\ln (2) / \ln (3)=0.667$. It means that the Cantor dust has an intermediate dimension between a point and a line. According to the definitions introduced by Carpinteri [25] such an object is an invasive point fractal or a lacunar line fractal.

Eq. (2) states that, if at each observation scale we properly change the specimen for the measure of the set with the law $\left(r_{k} L_{0}\right)^{d_{M}}$, then the measure of each pre-fractal and the dimension of fractal itself remain invariant. Other fractal sets with different dimensions may be found in literature and it is now fully recognized that all dimensions $d_{M} \in \mathbb{R}^{+}$cover the voids in the dimensions of Euclidean space.

\section{The anomalous transport across fractal media}

Let us assume that the flux of a Newtonian fluid occurs in a fractal-like three-dimensional porous media with anomalous dimension of the cross sectional area $\left[A_{F}\right]=L^{d}$ where $1 \leqslant d \leqslant 2$ is the Hausdorff dimension of the fractal set.

The overall volume of the porous solid is assumed as a subset of the 3 d Euclidean volume $V_{E}$ that is $V_{F} \subseteq V_{E}$ with dimension $\left[V_{F}\right]=L^{1+d}$. If, instead, the usual Euclidean measure is used, then the cross-section area measure $A_{F}=0$ and the volume measure $V_{F}=0$.

With reference to Fig. 2, let us assume that a coordinate system $\left\{0, x_{1}, x_{2}, x_{3}\right\}$ is attached to the Euclidean volume surrounding $V_{F}$ and let us define $l$ and $h$, respectively, the Euclidean length of the solid and the Euclidean measure of a characteristic dimension of the generator. Under these circumstances the cross-sectional area and the volume measure of the

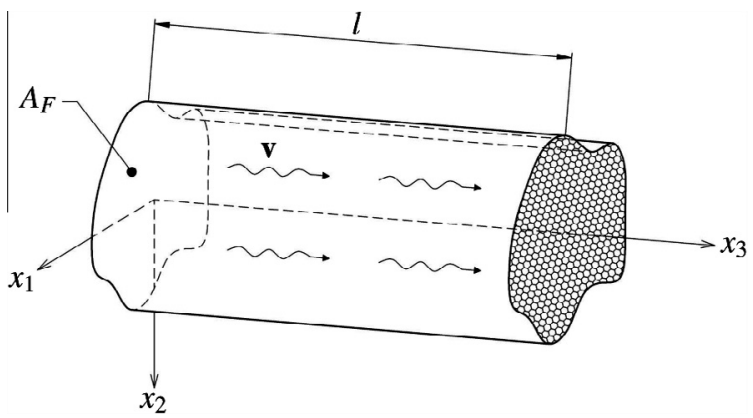

Fig. 2. Laminar flow in porous media. 
medium reads, respectively, $A_{F} \propto h^{d}$ and $V_{F} \propto h^{d} l$ with an appropriate proportionality coefficient that depends on the shape of the cross-sectional area.

Let us assume that the velocity field of the moving fluid across the material pores is $\mathbf{v}^{T}\left(x_{1}, x_{2}, x_{3}\right)=[0,0, v(\mathbf{x}, t)]$ where $\mathbf{x}^{T}=\left[x_{1}, x_{2}, x_{3}\right]$ is the coordinate vector. The gravity is neglected for the considered motion and the driving force of the moving fluid is the pressure gradient $\Delta p(t)\left([\Delta p(t)]=\mathrm{kg} \mathrm{m}^{-1} \mathrm{~s}^{-2}\right)$ that is maintained in range with values of the Reynolds number $R e=\rho v \delta / \mu$ lower than the value corresponding to the transition regime for the assumed fluid motion. In the expression of the Reynolds number $\rho$ is the density of the fluid $\left([\rho]=\mathrm{kg} \mathrm{m}^{-3}\right), \delta$ is the hydraulic diameter $([\delta]=m)$ and $\mu$ is the dynamic viscosity $\left([\mu]=\mathrm{kg} \mathrm{m}^{-1} \mathrm{~s}^{-1}\right)$. All the physical quantities will be expressed in S.I. units.

In passing we remark that fractal porous materials possess a power-law scaling of some geometrical features. A different scenario is observed with materials with power-law scaling of physical properties as functionally graded materials. It may be observed that media with power-law scaling may be heterogeneous materials or not, and in this latter case, a fractal geometry may be underlined.

\subsection{Discrete resolution scale approach}

In this study we confine our analysis to self-similar fractal sets, that are fractals without any shape changes with the resolution scale that is dubbed $k$ and represents the discrete sequence $k=0,1,2, \ldots, \infty$; in this setting the cross-sectional area at the $k$ th resolution scale, namely $A_{F}^{(k)}$, may be expressed as:

$$
A_{F}^{(k)}=\xi_{F} n_{k}\left(\frac{h}{\chi_{k}}\right)^{2}=\xi_{F}\left(\frac{h}{a^{f_{k}}}\right)^{2} b^{g_{k}}
$$

where $\xi_{F}$ is a shape factor that depends on the shape of the self-similar object, $n_{k}=b^{g_{k}}$ is the number of pre-fractal at resolution $k, \chi_{k}=a^{f_{k}}$ is the scale resolution factor of the $k$ th pre-fractal that is the division factor of the $k$ th length to yield an object self similar to the parent set and $a, b, f_{k}$ and $g_{k}$ depend on the fractal construction rule; for instance, the Sierpinski Carpet yields $a=3, b=2, f_{k}=k$ and $g_{k}=3 k$ (see Section 3.1.1).

The evaluation of the cross-sectional area in the Euclidean metric in Eq. (3) is not invariant at any resolution scale and, as the invariance measure relation is imposed to the Mandelbrot dimension, we get:

$$
\xi_{F}\left(\frac{h}{a^{f_{k}}}\right)^{d} b^{g_{k}}=\xi_{F} h^{d}
$$

yielding the Mandelbrot dimension of the set as:

$$
d=d_{M}=\frac{g_{k}}{f_{k}} \frac{\ln (b)}{\ln (a)}, \quad k=0,1,2, \ldots, \infty
$$

As we assume a self-similar fractal set, without any non-linear transformations among different scales, the geometric dependent exponents $f_{k}$ and $g_{k}$ in Eqs. (3) and (4) may be defined as appropriate linear functions of the resolution level $k$, as:

$$
f_{k}=r k, \quad g_{k}=s k
$$

where $r, s \in \mathbb{N}-\{0\}$ are the resolution factors.

The lack of the geometric invariance of the Euclidean metric yields that the outgoing flux across the different channels of the porous medium is not uniform at any resolution scale. This consideration yield that the overall outgoing flux is provided by the algebraic contribution of the outgoing fluxes at $x_{3}=l$ from the channels with cross-sections represented by the material pores as:

$$
q(t)=\sum_{k=0}^{\infty} q_{k}(t)=\sum_{k=0}^{\infty} A_{F}^{(k)} \bar{v}_{k}(t)
$$

where $\bar{v}_{k}(l, t)$ is the mean velocity field in the $k$ th pore dimension that is uniform along the pore channels and it will be denoted hereinafter, disregarding the spatial dependence, as $\bar{v}_{k}(t)$.

In the following we prefer to introduce non-dimensional flux, defined as $\bar{q}(t)=q(t) / q_{E}(t)$ that corresponds to the outgoing flux from the fractal porous media referred to the flux across an Euclidean cross-section $A_{E}$ embedding the fractal crosssection $A_{F}$.

In this setting the non-dimensional flux $\bar{q}(t)$ may be obtained from Eq. (7) as

$$
\bar{q}(t)=\frac{q(t)}{q_{E}(t)}=\sum_{k=0}^{\infty} \frac{q_{k}(t)}{q_{E}(t)}=\sum_{k=0}^{\infty} \frac{A_{F}^{(k)} \bar{v}_{k}(t)}{A_{E} \bar{v}_{E}(t)}
$$

where we denoted $\bar{v}_{E}(t)$ the mean velocity field due to the pressure gradient $J=\Delta p / l$ across the Euclidean cross-section $A_{E}$, under the assumption of linear viscous fluid and small Reynolds number that read $\bar{\nu}_{E}(t)=\alpha_{s} \alpha_{r} J h^{2} a^{2 r} / \mu: \alpha_{s}$ is an appropriate dimensionless coefficient depending upon the shape of the channel cross-section and Ris the scale-dependent hydraulic 
radius of the fluid channel. The hydraulic radius may be always expressed as proportional to the characteristic dimensions (base, height) of the cross-section by a proper dimensionless radius factor $\alpha_{r}$ as:

$$
R=\frac{h \sqrt{\alpha_{r}}}{a^{r k}}
$$

The mean velocity field across the $k$ th pore size may be expressed in terms of the velocity $\bar{v}_{E}(t)$ as:

$$
\bar{v}_{k}(t)=\bar{v}_{E}(t) \frac{1}{a^{2 r(k+1)}}
$$

yielding for the non-dimensional flux $\bar{q}(t)$ in Eq. (8) the relation:

$$
\bar{q}(t)=\frac{1}{a^{4 r}} \sum_{k=0}^{\infty}\left(\frac{b^{s}}{a^{4 r}}\right)^{k}
$$

since the ratio $A_{F}^{(k)} / A_{E}=b^{s k} / a^{2 r(k+1)}$.

Let us restrict our attention to the flow rate that leaves the porous solid at time $t$, namely $q_{L}(t)$. To this aim we observe that the contribution of the fluid contained in the $k$ th pore, namely $q_{k}(t)$, must be considered up to times $t_{k}$ as

$$
t_{k}=\left|\frac{l}{\bar{v}_{k}}\right|=\left|\frac{l}{\bar{v}_{E}}\right| a^{2 r(k+1)}=t_{E} a^{2 r(k+1)}
$$

where $t_{E}$ is the time involved by fluid columns contained in volume $V_{E}$ to arrive at abscissa $x_{3}=l$. Under these circumstances the effective volume time rate reaching abscissa $x_{3}=l$ of the fluid volume in $V_{F}$ is obtained as:

$$
\bar{q}_{L}(t)=\frac{q_{L}(t)}{q_{E}(t)}=\sum_{k=0}^{\infty} \frac{q_{k}(t) H\left(t_{k}-t\right)}{q_{E}(t)}=\frac{1}{a^{4 r}} \sum_{k=0}^{\infty}\left(\frac{b^{s}}{a^{4 r}}\right)^{k} H\left(t_{k}-t\right)
$$

where $H(x)$ is the unit step function defined as:

$$
H(x)=\left\{\begin{array}{lll}
1 & \text { if } & x>0 \\
0 & \text { if } & x \leqslant 0
\end{array}\right.
$$

Substituting the unit step function in Eq. (14) in Eq. (13), the non-dimensional outgoing volume rate $\bar{q}_{L}(t)$ reads:

$$
\bar{q}_{L}(t)=\left\{\begin{array}{lll}
\frac{1}{a^{4 r}-b^{s}}\left(\frac{b^{5}}{a^{4}}\right)^{0} & \text { if } & 0 \leqslant t<t_{0} \\
\frac{1}{a^{4 r}-b^{5}}\left(\frac{b^{5}}{a^{4}}\right)^{1} & \text { if } & t_{0} \leqslant t<t_{1} \\
\cdots & & \\
\frac{1}{a^{4 r}-b^{s}}\left(\frac{b^{5}}{a^{4}}\right)^{n} & \text { if } & t_{n-1} \leqslant t<t_{n}
\end{array}\right.
$$

the expression of the outgoing flux in Eq. (15) shows that it does not change in any time intervals $\left[t_{n-1}, t_{n}\right], n=0,1,2, \ldots, \infty$ (where we set $t_{-1}=0$ ) with value:

$$
\bar{q}_{L}^{(n)}=\frac{1}{a^{4 r}-b^{s}}\left(\frac{b^{s}}{a^{4 r}}\right)^{n}=\lambda\left(\frac{b^{s}}{a^{4 r}}\right)^{n}
$$

and the non-dimensional coefficient $\lambda$ depends on the geometric rule of fractal definition.

The rate of change of the outgoing flux $\bar{q}_{L}(t)$ may be obtained as explicit function of time tas we consider logarithm of Eq. (12) and (16) as

$$
\begin{aligned}
& \ln \left[t_{n}\right]=\ln \left[t_{E}\right]+2 r(n+1) \ln [a] \\
& \ln \left[\bar{q}_{L}^{(n)}\right]=\ln [\lambda]+n(s \ln [b]-4 r \ln [a])
\end{aligned}
$$

that may be solved yielding, after some straightforward algebra:

$$
\bar{q}_{L}(t)=\lambda\left(\frac{t}{t_{0}}\right)^{-\beta}, \quad t>t_{0}=t_{E} a^{2 r}
$$

and

$$
\beta=2-\frac{s \ln (b)}{2 r \ln (a)}=2-\frac{d}{2}
$$


the variation of the outgoing flux is therefore described by:

$$
\bar{q}_{L}(t)=\left\{\begin{array}{lll}
\lambda & \text { if } & 0<t \leqslant t_{0} \\
\lambda\left(\frac{t}{t_{0}}\right)^{-\beta} & \text { if } & t>t_{0}
\end{array}\right.
$$

Eq. (20) corresponds to a power-law relation among the outgoing flux and the observer time and some examples of the effective outgoing flux from a fractal porous media have been reported in the next sections.

The limiting cases of Eq. (20) are relative to geometric integer dimension, namely $d=2$ and $d=1$, of the cross-section measure. In more details the cases $d=2$ and $d=1$ involve identical features of the cross-section geometry at any resolution scale. In such cases the number of subdivision $b^{\text {sk }}$ should be equal to the scaling factor $a^{r k}$ in power $d$ at any resolution scale $k$ :

$$
b^{s k}=a^{r k d}, \quad \forall k \in \mathbb{N} \Rightarrow b^{s}=a^{r d}
$$

For $d=2$ from Eq. (19) we obtain $\beta=1$ then we have

$$
\bar{q}_{L}(t)=\left\{\begin{array}{lll}
\lambda & \text { if } & 0<t \leqslant t_{0} \\
\lambda\left(\frac{t}{t_{0}}\right)^{-1} & \text { if } & t>t_{0}
\end{array}\right.
$$

Similarly, for the case $d=1$, from Eq. (19) we obtain $\beta=3 / 2$ then the expression of the flux in Eq. (20) may be rewritten as

$$
\bar{q}_{L}(t)= \begin{cases}\lambda & \text { if } \quad 0<t \leqslant t_{0} \\ \lambda\left(\frac{t}{t_{0}}\right)^{-\frac{3}{2}} & \text { if } t>t_{0}\end{cases}
$$

The limiting cases of Euclidean cross-sections, namely $d=2$ and $d=1$, correspond to time scales $t^{-1}$ and $t^{-\frac{3}{2}}$ respectively. The case of anomalous time scaling of the outgoing flux ma be obtained, instead, with a power law scaling of the geometric measure of the solid cross-section yielding, in this case, $1<\beta<3 / 2$. Some examples of power-law scaling of the outgoing flux across a fractal cross-section will be introduced in the next section.

\subsubsection{Anomalous time variation across a Sierpinski carpet-like fractal}

Let us consider a porous solid consisting of a parallelepiped with a square section of side $3 h$ and length $l$ : let us divide the section in 9 equal squares of side $h$ and subtract to the initial section the central square. By repeating this operation many times we will get the fractal shown in Fig. 4, in which channels are obtained by iterating for three times the procedure described.

We want to study the outflow from the porous medium: at the initial time the fluid is at rest and the pressure $p$ is the same in both sections $S 1_{1}$ and $S_{2}$ which are the only permeable walls as shown in Fig. 3 . For $t>0$ we have that the pressure $p_{2}$ in the cross-section $S_{2}$ becomes less than the pressure $p_{1}$ in the cross-section $S_{1}$ and these values are kept constant over time: it will therefore have a fluid motion from section $S_{1}$ to section $S_{1}$ and we want to evaluate the time course of the nondimensional flow $\bar{q}_{L}(t)$ through Section 2 .

In particular, referring to Fig. 3 , as regards the dimension of the side we will have: $h_{0}=h, h_{1}=h / 3, h_{2}=h / 9, \ldots$, $h_{k}=h / 3^{k}$ and in accordance with relation (3), noting that the total number of pre-fractal at scale resolution $k=0,1,2, \ldots, \infty$ is $2^{3 k}$ we have

$$
A_{F}^{(k)}=\xi_{F}\left(\frac{h}{a^{r k}}\right)^{2} b^{s k}=\left(\frac{h}{3^{k}}\right)^{2} 2^{3 k}
$$

from which we derive that $\xi_{F}=1$ and the relations $a=3, r=1, b=2$ and $s=3$.

Considering the definition of hydraulic radius $R=A / P$ where $A$ and $P$ are respectively the area and the perimeter of the cross section and taking into account relation (9), the coefficient $\alpha_{r}$ can be evaluated as:

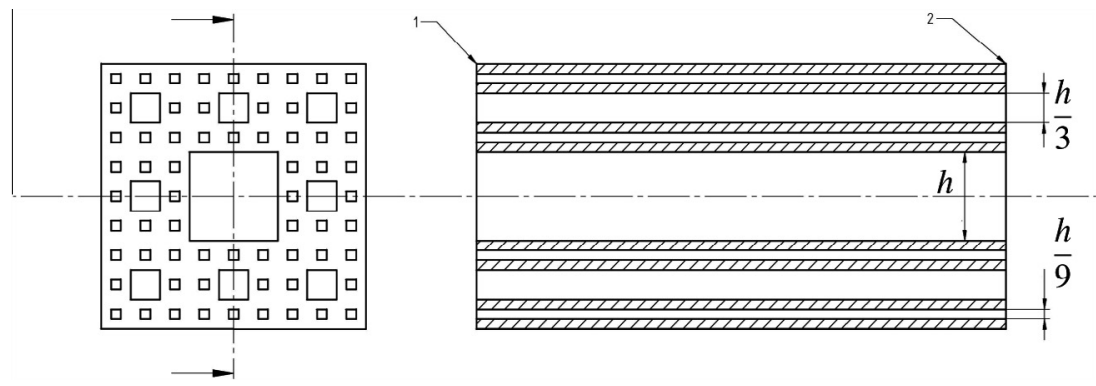

Fig. 3. Views and longitudinal section of the Sierpinski carpet-like fractal. 


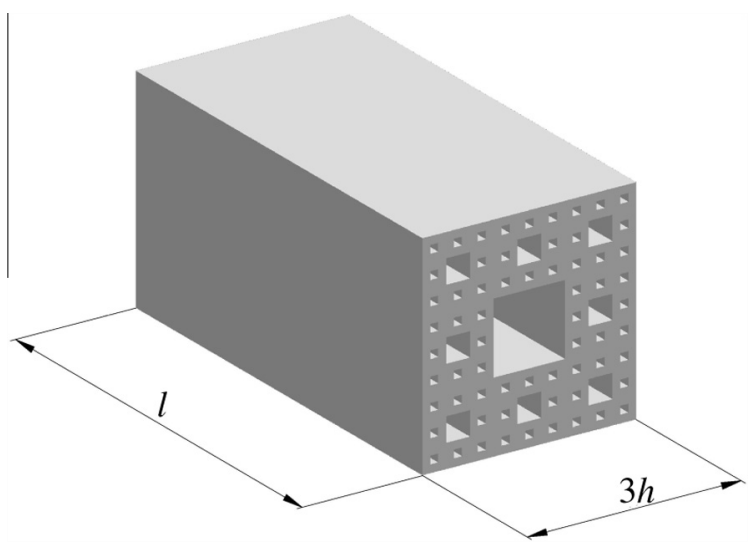

Fig. 4. Sierpinski carpet-like fractal.

$$
\alpha_{r}=R^{2}\left(\frac{a^{r k}}{h}\right)^{2}=\left(\frac{h}{4 a^{r k}}\right)^{2}\left(\frac{a^{r k}}{h}\right)^{2}=\frac{1}{16}
$$

Finally, from Eq. (5) we can evaluate the fractal dimension $d$ of the cross section

$$
d=\frac{s \ln (b)}{r \ln (a)}=\frac{3 \ln (2)}{\ln (3)} \simeq 1.89
$$

and from Eq. (19) the decaying exponent $\beta$ as:

$$
\beta=2-\frac{d}{2} \simeq 1.05
$$

The flow along the time as expressed by Eq. (20) is similar to that shown in Fig. 6 .

\subsubsection{Anomalous time variation across a Sierpinski gasket-like fractal}

A further application concerns the porous medium defined by the Sierpinski gasket-like fractal (Fig. 5) i.e. from fractal obtained starting from an equilateral triangle to which we subtract triangles of decreasing side by a factor $1 / 2$ and subject to the same boundary conditions, namely pressure gradient $J$ kept constant over time.

The procedure used to evaluate the flow over time is the same as the one followed in the previous paragraph; it should be noted that in each successive iteration the side $h$ of the largest outer triangle is halved, then we can write that $h_{k}=h / 2^{k}$.

The total number of pre-fractal at scale resolution $k=0,1,2, \ldots, \infty$ is $3^{k}$ and in accordance with relation (3), the cross sectional area at the scale resolution $k$ reads

$$
A_{F}^{(k)}=\xi_{F}\left(\frac{h}{a^{r k}}\right)^{2} b^{s k}=\frac{\sqrt{3}}{4}\left(\frac{h}{2^{k}}\right)^{2} 3^{k}
$$

from Eq. (28) we obtain all the parameter depending on the construction rule of the fractal, namely: $\xi_{F}=\sqrt{3} / 4, a=2, r=1, b=3$ and $s=1$ and taking into account relation (9), the coefficient $\alpha_{r}$ can be evaluated as:

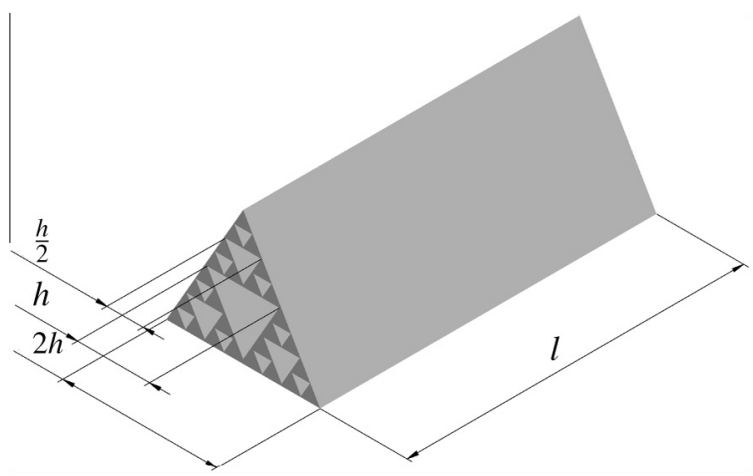

Fig. 5. Sierpinski gasket-like fractal. 


$$
\alpha_{r}=R^{2}\left(\frac{a^{r k}}{h}\right)^{2}=\left(\frac{h}{4 \sqrt{3} a^{r k}}\right)^{2}\left(\frac{a^{r k}}{h}\right)^{2}=\frac{1}{48}
$$

and from Eq. (5) we can evaluate the fractal dimension $d$ of the cross section

$$
d=\frac{s \ln (b)}{r \ln (a)}=\frac{\ln (3)}{\ln (2)} \simeq 1.58
$$

and from Eq. (19) the decaying exponent $\beta$ as:

$$
\beta=2-\frac{d}{2} \simeq 1.21
$$

in Fig. 6 it can be seen the graph of the relationship (18) related to this case.

The existence of a power-law scaling of the outgoing flux of the fluid mass initially contained in the fractal pores of the medium may also be related with the non-dense curdling of the reachable states of a linear physical system $W(t)$ with the normalized fractal scaling of the memory function $[21,16]$. In that context a relation of the type

$$
W(t)=A_{d} D^{-d} f(t)=\frac{A_{d}}{t^{-d}} \frac{1}{\Gamma(d)} \int_{0}^{t}(t-\tau)^{d-1} f(\tau) d \tau
$$

with $A_{d}$ a proper normalization constant and $f(t)$ the forcing function has been obtained for $0<d<1$, being $d$ the dimension of the fractal set.

The approximation involved in Eq. (32) is due to the need of an appropriate homogenization of the fractal set of the physical state of the system by means of their continuous-type Hausdorff-dimension. Moreover, the presence of a convolution integral for the reachable state of the physical system involves the existence of a linear cause-effect relation.

The physical system presented in the paper, however, does not obey to this requirement as it has been observed in Eq. (20) since the provided outgoing flux does not depend, linearly, of the applied pressure gradient $J$.

In the next section we show that a fractional order generalization of laminar transport equation may be obtained for the proposed model of mass transport across a fractal porous media under the following main assumptions:

1. The existence of a continuum scaling coefficient $\varepsilon$ of self-similar pores of the considered fractal set.

2. An appropriate linearization of the force-flux relation around an initial value $J_{0}=\Delta p_{0} / l$ of the pressure gradient.

\subsection{Fractional-order generalization of laminar transport equations}

The non-dimensional outgoing flux in Eq. (7) may be reverted in a continuous form as we observe that, for real sets, there is a continuous variation among various resolution scale. In the following the scaling resolution is dubbed $\varepsilon$ and it represents the real number corresponding the continuation of the discrete sequence $k=0,1,2, \ldots, \infty$ on the real axis.

Under these circumstances the geometric dependent exponents $f_{k}$ and $g_{k}$, defined by Eq. (6) may be cast in their continualized counterpart $f_{k} \rightarrow f(\varepsilon)$ and $g_{k} \rightarrow g(\varepsilon)$ as:

$$
f(\varepsilon)=r \varepsilon, \quad g(\varepsilon)=s \varepsilon
$$

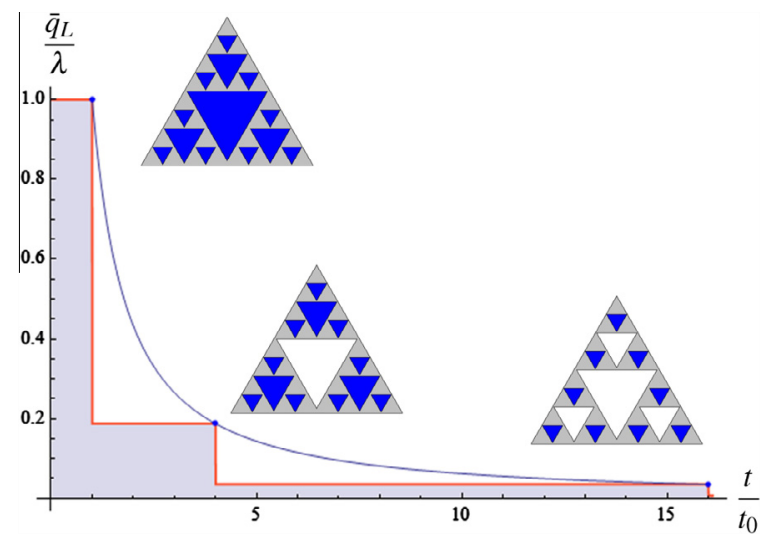

Fig. 6. Graph of the non-dimensional flow $\bar{q}_{L} / \lambda$ along the non-dimensional time $t / t_{0}$ for the Sierpinski gasket-like fractal. A similar trend is observed in the case of the Sierpinski carpet-like fractal examined in previous section. 
where $r$ and sare the resolution factors as defined in Eq. (6). The ratio of the cross-sectional area at resolution scale $\varepsilon$, namely $A_{F}(\varepsilon) / A_{E}$ reads:

$$
\frac{A_{F}(\varepsilon)}{A_{E}}=\left(\frac{b^{s}}{a^{2 r}}\right)^{\varepsilon} \frac{1}{a^{2 r}}
$$

whereas for the ratio of the mean velocity field, namely $\bar{v}(\varepsilon) / v_{E}=a^{-2 r(\varepsilon+1)}$ yielding, for the non-dimensional flux across the fractal porous solid

$$
\bar{q}(t)=\frac{q(t)}{q_{E}}=\lim _{\Delta q_{k} \rightarrow 0} \sum_{k=0}^{\infty} \frac{\Delta q_{k}(t)}{q_{E}}=\int_{0}^{\infty} d\left(\frac{q(\varepsilon)}{q_{E}}\right)=\int_{0}^{\infty} d\left(\frac{A_{F}(\varepsilon) \bar{v}(\varepsilon)}{A_{E} v_{E}}\right)
$$

Similarly to the case of the geometrical ideal fractals discussed in Section 3.1, the volume of fluid that leaves the porous solid at the time instant $t$ is $\bar{q}_{L}(t)$ that may be evaluated as:

$$
\bar{q}_{L}(t)=\int_{0}^{\infty} H\left(t_{\varepsilon}-t\right) d\left(\frac{A_{F}(\varepsilon) \bar{v}(\varepsilon)}{A_{E} v_{E}}\right)
$$

Eq. (36) may be further simplified as we evaluate, for each scale $\varepsilon$, the time $t_{\varepsilon}$ at which the fluid columns at scale $\varepsilon$, entering from the left side of the volume at $x_{3}=0$, reaches the right side at $x_{3}=l$ as:

$$
t_{\varepsilon}=\left|\frac{l}{\bar{v}(\varepsilon)}\right|=t_{E} a^{2 r(\varepsilon+1)}
$$

that represents the continualized counterpart of relation (12); Eq. (37) may be used to evaluate the highest scale resolution $\bar{\varepsilon}$ to be included in the measured outgoing flux at time $t$, namely $\bar{q}_{L}(t)$, as from Eq. (37):

$$
\bar{\varepsilon}=\ln \left[\frac{t_{\varepsilon}}{t_{E}}\right] \frac{1}{\ln \left[a^{2 r}\right]}
$$

yielding for Eq. (36)

$$
\bar{q}_{L}\left(t_{\varepsilon}\right)=\int_{\bar{\varepsilon}}^{\infty}\left[\frac{d A_{F}(\varepsilon)}{d \varepsilon} \frac{\bar{v}(\varepsilon)}{v_{E}}+\frac{1}{v_{E}} \frac{d \bar{v}(\varepsilon)}{d \varepsilon} \frac{A_{F}(\varepsilon)}{A_{E}}\right] d \varepsilon
$$

yielding, upon proper integrations:

$$
\bar{q}_{L}\left(t_{\varepsilon}\right)=\frac{1}{a^{4 r}} \int_{\bar{\varepsilon}}^{\infty} \frac{d}{d \varepsilon}\left(\frac{b^{S \varepsilon}}{a^{4 r \varepsilon}}\right) d \varepsilon=\frac{1}{a^{4 r}}\left(\frac{b^{s}}{a^{4 r}}\right)^{\bar{\varepsilon}}
$$

that may be cast in explicit terms of time $t$ taking natural logarithms of Eq. (40) as:

$$
\ln \left[\bar{q}_{L}\right]=\bar{\varepsilon}(s \ln [b]-4 r \ln [a])-4 r \ln [a]
$$

yielding, upon substitution of Eq. (38)

$$
\ln \left[\bar{q}_{L}\right]=\ln \left[\frac{t_{\varepsilon}}{t_{E}}\right] \frac{s \ln [b]-4 r \ln [a]}{2 r \ln [a]}-4 r \ln [a]=\ln \left[\frac{t_{\varepsilon}}{t_{E}}\right]^{-\beta}-4 r \ln [a]
$$

with $\beta=2-d / 2$ as in previous section, yielding, for the outgoing flux as explicitly dependent of time $t_{\varepsilon} \longrightarrow t$

$$
\bar{q}_{L}(t)= \begin{cases}a^{2 r(\beta-2)} & \text { if } \quad 0<t \leqslant t_{0}=t_{E} a^{2 r} \\ a^{2 r(\beta-2)}\left(\frac{t}{t_{0}}\right)^{-\beta} & \text { if } \quad t>t_{0}=t_{E} a^{2 r}\end{cases}
$$

that may be expressed in terms of unit step function as:

$$
\bar{q}_{L}(t)=a^{2 r(\beta-2)}\left[H\left(t_{0}-t\right)+\left(\frac{t}{t_{0}}\right)^{-\beta} H\left(t-t_{0}\right)\right]=a^{2 r(\beta-2)}\left[H\left(t_{0}-t\right)+\lambda_{L}(\beta) t^{-\beta} J^{-\beta} H\left(t-t_{0}\right)\right]
$$

where the physical coefficient $\lambda_{L}(\beta)$ is:

$$
\lambda_{L}(\beta)=\left(\frac{\alpha_{s} \alpha_{r} h^{2}}{\mu l}\right)^{-\beta}
$$

The expression of the flux reported in Eq. (44) is quite similar to the well-known Nutting relation of viscoelasticity, namely

$$
u=A t^{n} F^{m}
$$

with $n$ and $m$ real number, $F$ the applied force traction and $u$ the displacement of the tested specimen. The formal analogy among Eq. (44) and Eq. (46) leads to conclude that introducing a proper linearization with respect to the pressure gradient $J$, 
a fractional-order laminar transport equation is involved. Indeed let us define the pressure field $J\left(x_{3}, t\right)$ as the sum of the non uniform (in the sense that it varies along the $x_{3}$ axis) mean field $J_{0}\left(x_{3}\right)$ that does not depend on the time $t$ and a space-time perturbation field $J_{1}\left(x_{3}, t\right)$ that varies in a small range around the value $J_{0}$.

The evolution of the pressure field in terms of $J_{0}\left(x_{3}\right)$ and $J_{1}\left(x_{3}, t\right)$ reads:

$$
J\left(x_{3}, t\right)=J_{0}\left(x_{3}\right)+J_{1}\left(x_{3}, t\right)
$$

Introducing Eq. (47) into Eq. (44) and using a Taylor series expansion up to the first term with respect to the mean value $J_{0}\left(x_{3}\right)$ it yields:

$$
\bar{q}_{L}\left(x_{3}, t\right) \simeq a^{2 r(\beta-2)}\left[H\left(t_{0}-t\right)+\lambda_{L}(\beta) t^{-\beta}\left(\left[J_{0}\left(x_{3}\right)\right]^{-\beta}-\beta\left[J_{0}\left(x_{3}\right)\right]^{-(\beta+1)} J_{1}\left(x_{3}, t\right)\right) H\left(t-t_{0}\right)\right], \quad t>0
$$

where we assumed that the pressure gradient fluctuation $J_{1}\left(x_{3}, t\right)$ is small with respect to the mean pressure gradient $J_{0}\left(x_{3}\right)$ according to the assumption of laminar flow.

The linearized version of Eq. (44) reported in Eq. (48) allows for the definition of a fractional-order laminar transport equation. To this aim we first evaluate the fluctuating non-dimensional flux $\bar{q}_{0}\left(x_{3}, t\right)$ due to $J_{1}\left(x_{3}, t\right)$ with respect to the non-fluctuating part induced by $J_{0}\left(x_{3}\right)$ that is:

$$
\begin{aligned}
\bar{q}_{0}\left(x_{3}, t\right) & =\bar{q}_{L}\left(x_{3}, t\right)-a^{2 r(\beta-2)}\left[H\left(t_{0}-t\right)+\lambda_{L}(\beta) t^{-\beta}\left[J_{0}\left(x_{3}\right)\right]^{-\beta} H\left(t-t_{0}\right)\right] \\
& =-a^{2 r(\beta-2)} \beta \lambda_{L}(\beta) t^{-\beta}\left[J_{0}\left(x_{3}\right)\right]^{-(\beta+1)} J_{1}\left(x_{3}, t\right) H\left(t-t_{0}\right)
\end{aligned}
$$

The knowledge of the outgoing flux $\bar{q}_{0}\left(x_{3}, t\right)$ due to the pressure increment $J_{1}\left(x_{3}, t\right)$ is useful to evaluate the flux $\bar{q}_{0}\left(x_{3}, t\right)$ due to a generic pressure gradient $J_{1}\left(x_{3}, t\right)$ by means of the convolution operator defined as:

$$
(f * g)(t)=\int_{-\infty}^{\infty} f(\tau) g(t-\tau) d \tau=\int_{-\infty}^{\infty} g(\tau) f(t-\tau) d \tau
$$

Indeed, in case of linear system, the outgoing flux $\bar{q}_{0}$ may be written as:

$$
\bar{q}_{0}(t)=\left(\dot{j}_{1} * c_{1}\right)(t)
$$

being $\dot{J}_{1}\left(x_{3}, t\right)=d J_{1} / d t$ and where we defined $c_{1}$ as:

$$
\left.c_{1}(t)=-a^{2 r(\beta-2)} \beta \lambda_{L}(\beta) t^{-\beta} J_{0}\left(x_{3}\right)\right]^{-(\beta+1)} H\left(t-t_{0}\right)
$$

On the other hand, considering that the function $t^{-\beta} H\left(t-t_{0}\right)$ in Eq. (52) may be rewritten as $t^{-\beta} H\left(t-t_{0}\right)=t^{-\beta} H(t)-t^{-\beta} H\left(t_{0}-t\right)$, as shown in Fig. 7, we obtain:

$$
\begin{aligned}
\left(\dot{J}_{1} * c_{1}\right)(t)= & -a^{2 r(\beta-2)} \beta \lambda_{L}(\beta)\left[J_{0}\left(x_{3}\right)\right]^{-(\beta+1)}\left[\Gamma(1-\beta)\left(\mathrm{D}_{0}^{\beta} J_{1}\right)(t)-J_{1}\left(x_{3}, 0\right) t^{-\beta}\right] H\left(t-t_{0}\right) \\
& +\frac{a^{2 r(\beta-2)} \beta \lambda_{L}(\beta)\left[J_{0}\left(x_{3}\right)\right]^{-(\beta+1)}}{1-\beta}\left[\dot{J}_{1}\left(x_{3}, t-t_{0}\right) t_{0}^{1-\beta}+\int_{t-t_{0}}^{t} \ddot{J}_{1}\left(x_{3}, \tau\right)(t-\tau)^{1-\beta} d \tau\right] H\left(t-t_{0}\right)
\end{aligned}
$$

yielding the outgoing flux in the form:

$$
\bar{q}_{0}\left(x_{3}, t\right)=a^{2 r(\beta-2)} \lambda_{L}(\beta)\left[J_{0}\left(x_{3}\right)\right]^{-(\beta+1)}\left[\beta^{2} \Gamma(-\beta)\left(\mathrm{D}_{0}^{\beta} J_{1}\right)(t)+\beta J_{1}\left(x_{3}, 0\right) t^{-\beta}\right] H\left(t-t_{0}\right)+R_{\beta}\left(x_{3}, t\right)
$$

where $R_{\beta}\left(x_{3}, t\right)$ is a time-varying residual term expressed by:

$$
R_{\beta}\left(x_{3}, t\right)=\frac{a^{2 r(\beta-2)} \beta \lambda_{L}(\beta)\left[J_{0}\left(x_{3}\right)\right]^{-(\beta+1)}}{1-\beta}\left[\dot{J}_{1}\left(x_{3}, t-t_{0}\right) t_{0}^{1-\beta}+\int_{t-t_{0}}^{t} \ddot{J}_{1}\left(x_{3}, \tau\right)(t-\tau)^{1-\beta} d \tau\right] H\left(t-t_{0}\right)
$$

that is vanishing as $t_{0} \longrightarrow 0$ or in presence of slowly fluctuating pressure gradient so that $\ddot{J}_{1}\left(x_{3}, t\right) \simeq 0$.

In previous studies it has been proved that the pressure-flux relation through a fractal porous medium is related to the pressure gradient by means of a Caputo fractional derivative [26]. In the latter work the authors provide the existence of a

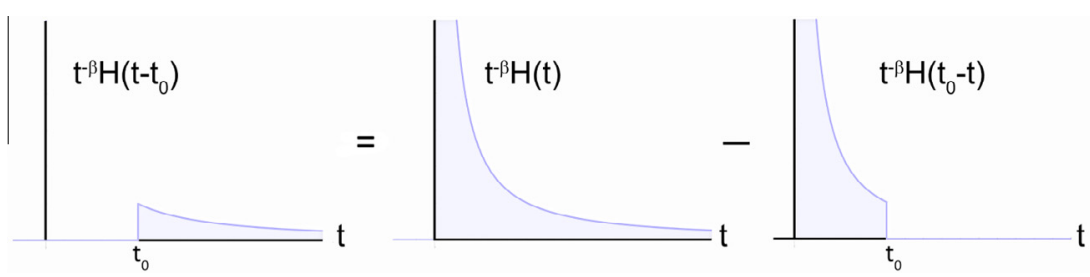

Fig. 7. Decomposition of the signal $t^{-\beta} H\left(t-t_{0}\right)$ as the difference between a power law $t^{-\beta} H(t)$ and the truncated power law $t^{-\beta} H\left(t_{0}-t\right)$. 
connection between fractal geometry and fractional calculus. Indeed it has been shown that the time evolution of a viscous fluid across a fractal porous medium is represented by a power-law. Furthermore, some focus have been provided about both the percolation and the seeping phenomena. In the case of the fluid seeping through the fractal porous medium under the effect of a pressure gradient, a fractional differential operator ruling the time evolution of the overall flux has been obtained by means of an appropriate linearization. However, it must be remarked that the relation in Eq. (24) in [26] strictly involves a convolution integral with a power-law kernel that is only formally analogous to a Caputo fractional derivative. Indeed, the presence of an exponent $1<\gamma<1.5$ yields an hyper-singular kernel that does not exactly correspond, from a mathematical perspective, to a Caputo fractional derivative [2].

Summing up, in this section the authors showed that as the flow of a Newtonian fluid in laminar flow across a fractal porous media is considered, then the time-decay of the outgoing flux of the fluid occupying the pores of the media is represented as a power-law of the time and the exponent of the power-law is related to the Mandelbrot dimension of the fractal media as from Eq. (54).

The flux is a non-linear function of the pressure gradient, similarly to the well-known relation involved in Nutting law for viscoelasticity and, under the assumption of small time fluctuation of the pressure gradient, then, a fractional-order generalization of laminar transport equation is obtained. Proper multidimensional generalization is actually underway and it will be published elsewhere.

Some 1D problems, in heat transfer and thermoelasticity, among others, that involve the use of the fractional-order calculus have been recently proposed [27-29]. Such problems, however, do not relate the order of the derivative to the geometric scaling of the medium. As an example, in a simple problem of poromechanics as the Terzaghi problem, the outgoing flux is described by the simple Darcy equation that is $q \propto \Delta p / l$; then the flux is proportional to the Oth-order derivative of the pressure gradient. With the proposed model the same governing equations of the Terzaghi seepage problem may be used introducing the relation in Eq. (54) for the force-flux relation.

\section{Conclusions}

In this paper the authors aim to introduce an ideal mechanical model leading to an anomalous time scaling of the state variables of the system. Such an approach yields a fractional-order generalization of laminar transport equation. In this regard the authors discuss the problem of a Newtonian fluid in the Poiseuille regime across a fractal porous media.

The evaluation of the overall outgoing flux of the fluid initially contained in the control volume yields a discrete-state physical system with curdled states distributed over a non-dense set of the real axis. This result is in totally agreement with discussions $[16,21,30,17]$ about the physical framework of fractional-order integrals obtained for linear-system in presence of discrete-state memory function.

In the present study it has been shown that the mechanics involved in the outgoing flux of a viscous fluid in a laminar regime across a fractal porous media is capable to describe the state curdling described in previous papers. However, the curdling is not accompanied by a linear force-flux relation and, therefore, the Boltzmann superposition principle, yielding fractional-order operators, can not be applied to the described mechanical system.

Fractional-order operators, instead, have been obtained under three main assumptions: (i) the existence of a continualized scale parameter $\varepsilon$ to describe the self-similar fractal pores of the media; (ii) a proper linearization of the non-linear force-flux relation around an initial value of the pressure gradient. As far as such assumptions have been introduced in the model, a fractional-order generalization of laminar transport equation is obtained in terms of the Riemann-Liouville fractional-order operators with differentiation order related to the fractal dimension of the porous set.

Extensions to high dimensions problems as well as to other forms of transport as Darcy/Fick diffusion is actually under way.

\section{Acknowledgments}

The authors are very grateful to Prof. Mario Di Paola for the precious time he dedicated to the discussion of the themes of this paper and wish to thank him for his support. Massimilano Zingales gratefully acknowledges the national research grant Prin 2010-2014 with national coordinator Prof. A. Luongo for the financial support.

\section{Appendix A. Hausdorff-Besitckovich measure}

The heuristic definition of the fractal dimension may be framed in a rigorous mathematical definition by introducing the Hausdorff-Besitckovich (HB) measure and dimension of a set $V$ (see [31] for details) denoted as $H^{S}(V)$ and $d_{H}$, respectively (set $V$ may be fractal or not).

Let us introduce for any set $V \subseteq \mathbb{R}^{m}$ a cover of the set $V$ as the union of subsets $G_{j} \subseteq \mathbb{R}^{m}, j=1,2, \ldots n$ such that $V \subseteq \bigcup_{j=1}^{n} G_{i}$ as shown in Fig. 8. Let us define the diameter of the subset $G_{j}$ of the set $\{G\}$ (union of the subsets $G_{j}$ ) as $\operatorname{diam}\left(G_{j}\right)=\left|G_{j}\right|=\sup \left\{|x-y|: x, y \in G_{j}\right\}$ and a $\delta$-cover of $V$, denoted as $\{G\}_{\delta}$ as a countable set $G_{j}:\left|G_{j}\right| \leqslant \delta$ with $j=$ $1,2, \ldots, m$ and $\delta \in \mathbb{R}^{+}$. The HB measure of the set $V$ in terms of a given $\delta$-cover is then represented as: 


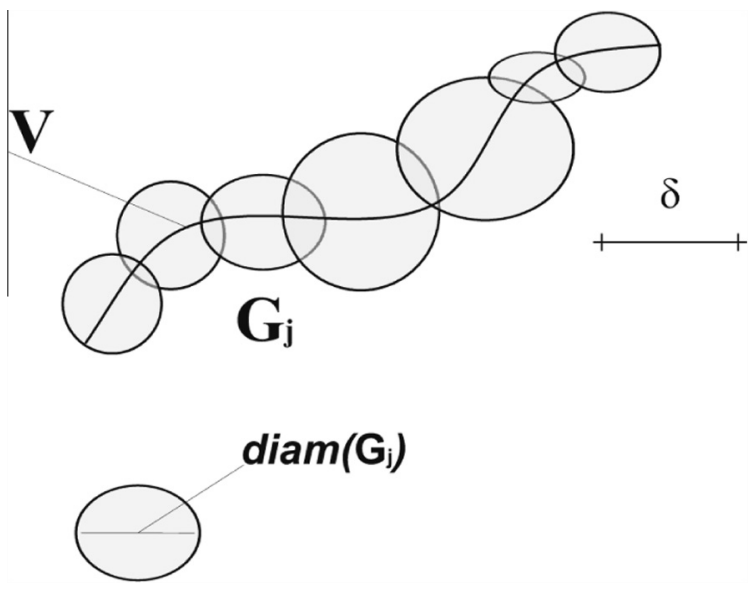

Fig. 8. Hausdorff-Besitckovich measure.

$$
H_{\delta}^{s}(V)=\inf \left\{\sum_{j=1}^{n}\left|G_{j}\right|^{s}: G_{j} \in\{G\}_{\delta}\right\}
$$

with $s \in \mathbb{R}^{+}$. The HB measure for the set $V$, denoted as $H^{s}(V)$ is represented as $H^{s}(V)=\lim _{\delta \rightarrow 0} H_{\delta}^{s}(V)$ and it strongly depends on values of the parameter $s$. As in fact it may be either zero or infinity with the exception of a single value of $s$, say $s^{*}$ such that $H^{s^{*}}(V)$ remains finite. Such a value $s^{*}$ is the HB dimension, denoted as $d_{H}$ that is:

$$
d_{H}=s^{*}=\inf \left\{s \geqslant 0: H^{s}(V)=0\right\}=\sup \left\{s \geqslant 0: H^{s}(V)=\infty\right\}
$$

An immediate meaning of Eq. (A.2) is that, as we want to measure a line by isolated points we need of infinite points, and if we want to measure a line by means of a surface we need a surface with vanishing area and so on. Therefore it may be concluded that only by using appropriate tool to measure an object we may define the appropriate dimension of the object itself. For the fractal Cantor set it may be easily recognized that the HB dimension exactly coalesces with $d_{M}$.

Moreover $d_{M}=d_{H}$ for all fractal sets built upon assigned laws, but in general, for an assigned fractal whose construction rules are not a priori known, the HB definition may be useful to obtain fractal dimension. Now we may proper define fractals as objects whose $\mathrm{HB}$ dimension $d_{H} \in \mathbb{R}^{+}$and preserving some invariance at any resolution scale.

\section{Appendix B. Basic definitions of fractional-order calculus}

Fractional calculus may be considered the extension of the ordinary differential calculus to non-integer powers of derivation orders (e.g. see [32,2]). In this section we address some basic notions about this mathematical tool.

The Euler-Gamma function $\Gamma(z)$ may be considered as the generalization of the factorial function since, as $z$ assumes integer values as $\Gamma(z+1)=z$ ! and it is defined as the result of the integral as follows:

$$
\Gamma(z)=\int_{0}^{\infty} e^{-x} x^{z-1} d x .
$$

The Riemann-Liouville fractional integral of arbitrary order $\beta>0$ of a function $f(t)$ has the following forms:

$$
\left(\mathrm{I}_{a}^{\beta} f\right)(t)=\frac{1}{\Gamma(\beta)} \int_{a}^{t}(t-\tau)^{\beta-1} f(\tau) d \tau
$$

while the Riemann-Liouville fractional derivative of order $\beta$ where $n-1 \leqslant \beta<n$ with $n \in \mathbb{N}$ is defined as

$$
\left(\mathrm{D}_{a}^{\beta} f\right)(t)=\frac{1}{\Gamma(n-\beta)} \frac{d^{n}}{d t^{n}} \int_{a}^{t} f(\tau)(t-\tau)^{n-\beta-1} d \tau
$$

The Riemann-Liouville fractional integrals and derivatives with $0<\beta<1$ of functions defined over intervals of the real axis, namely $f(t)$ such that $t \in[a, b] \subset \mathbb{R}$, have the following forms:

$$
\begin{aligned}
& \left(\mathrm{I}_{a}^{\beta} f\right)(t)=\frac{1}{\Gamma(\beta)} \int_{a}^{t} \frac{f(\tau)}{(t-\tau)^{1-\beta}} d \tau \\
& \left(\mathrm{D}_{a}^{\beta} f\right)(t)=\frac{f(a)}{\Gamma(1-\beta)(t-a)^{\beta}}+\frac{1}{\Gamma(1-\beta)} \int_{a}^{t} \frac{f^{\prime}(\tau)}{(t-\tau)^{\beta}} d \tau
\end{aligned}
$$


Beside Riemann-Liouville fractional operators defined above, another class of fractional derivative that is often used in the context of fractional viscoelasticity is represented by Caputo fractional derivatives defined as:

$$
\left({ }_{c} \mathrm{D}_{a}^{\beta} f\right)(t)=\frac{1}{\Gamma(n-\beta)} \int_{a}^{t} \frac{d^{n} f(\tau)}{d t^{n}}(t-\tau)^{n-\beta-1} d \tau
$$

being $n-1<\beta<n$. Whenever $0<\beta<1$ it reads as follows:

$$
\left({ }_{c} \mathrm{D}_{a}^{\beta} f\right)(t)=\frac{1}{\Gamma(1-\beta)} \int_{a}^{t} \frac{f \prime(\tau)}{(t-\tau)^{\beta}} d \tau
$$

A closer analysis of Eq. (B.5) and Eq. (B.7) shows that Caputo fractional derivative coincides with the integral part of the Riemann-Liouville fractional derivative in bounded domain. Moreover, the definition in Eq. (B.6) implies that the function $f(t)$ has to be absolutely integrable of order $m$ (e.g. in (B.7) the order is $m=1$ ). Whenever $f(a)=0$ Caputo and RiemannLiouville fractional derivatives coalesce. Similar considerations hold true also for Caputo and Riemann-Liouville fractional derivatives defined on the entire real axis.

Caputo fractional derivatives may be consider as the interpolation among the well-known integer-order derivatives, operating over functions $f(\circ)$ that belong to the class of Lebesgue integrable functions $\left(f\left(^{\circ}\right) \in L^{1}\right)$; as a consequence, they are very useful in the mathematical description of complex system evolution.

We recall that the Laplace integral transform is defined as follows:

$$
\mathscr{L}[f(t)]=\tilde{f}(s)=\int_{0}^{\infty} f(t) \mathrm{e}^{-s t} d t
$$

with $\operatorname{Re}(s)>a$. The function $f(t)$ may be restored from Laplace domain utilizing the inverse Laplace transform:

$$
f(t)=\mathscr{L}^{-1}[\tilde{f}(s)]=\int_{c-j \infty}^{c+j \infty} \tilde{f}(s) e^{s t} d s
$$

where $c=\operatorname{Re}(s)>a$. It is worth introducing integral transforms for Riemann-Liouville fractional operators and, similarly to classical calculus, the Laplace integral transform $\mathscr{L}(\circ)$ is defined in the following forms:

$$
\begin{aligned}
& \mathscr{L}\left[\left(\mathrm{D}_{0}^{\beta} f\right)(t)\right]=s^{\beta} \tilde{f}(s)-\sum_{k=0}^{n-1} s^{k}\left[\left(\mathrm{D}_{0}^{\beta-k-1} f\right)(t)\right]_{t=0} \\
& \mathscr{L}\left[\left(\mathrm{I}_{0}^{\beta} f\right)(t)\right]=s^{-\beta} \tilde{f}(s)
\end{aligned}
$$

being $n-1 \leqslant \beta<n$ for the fractional derivative. In a similar way, the Laplace transform of the Caputo fractional derivative is defined as

$$
\mathscr{L}\left[\left({ }_{c} \mathrm{D}_{0}^{\beta} f\right)(t)\right]=s^{\beta} \tilde{f}(s)-\sum_{k=0}^{n-1} s^{n-k-1}\left[\frac{d^{k} f(t)}{d t^{k}}\right]_{t=0}
$$

\section{References}

[1] Le Méhaute A, Crepy G. Introduction to transfer and motion in fractal media: the geometry of kinetics. Solid State Ionics 1983;9-10:17-30.

[2] Podlubny I. Fractional differential equations. Academic Press; 1993.

[3] Glöckle WG, Nonnenmacher TF. A fractional calculus approach to self-similar protein dynamics. Biophys J 1995;68(1):46-53.

[4] Magin RL, Ovadia M. Modeling the cardiac tissue electrode interface using fractional calculus. J Vib Control 2008;14(9-10):1431-42.

[5] Carpinteri A, Cornetti P, Kolwankar KM. Calculation of the tensile and flexural strength of disordered materials using fractional calculus. Chaos Solitons Fract 2004;21(3):623-32.

[6] Deseri L, Zingales M. A mechanical picture of fractional order Darcy equation. Commun Nonlinear Sci Numer Simul 2015;20:940-49.

[7] Di Paola M, Zingales M. Exact mechanical models of fractional hereditary materials. J Rheol 2012;56(5).

[8] Di Paola M, Pinnola FP, Zingales M. A discrete mechanical model of fractional hereditary materials. Meccanica 2013;48(7):1573-86.

[9] Tarasov V. Continuous medium model for fractal media. Phys Lett A 2005;336:167-74.

[10] Alexander S, Orbach R. Density of states on fractals: fractions. Le J Phys - Lett 1982;43:625-31.

[11] Mongioví MS, Zingales M. A non-local model of thermal energy transport: the fractional temperature equation. Int J Heat Mass Transfer 2013;67:593-601.

[12] Deseri L, Owen D. Invertible structured deformations and the geometry of multiple slip in single crystals. Int J Plast 2002;18:833-49.

[13] Deseri L, Owen D. Submacroscopically stable equilibria of elastic bodies undergoing disarrangements and dissipation. Math Mech Solids 2010;15:611-38.

[14] Tarasov V. Flow of fractal fluid in pipes: non-integer dimensional space approach. Chaos Solitons Fract 2014;67:26-37.

[15] Deseri L, Di Paola M, Zingales M, Pollaci P. Power-law hereditariness of hierarchical fractal bones. Int J Numer Methods Biomed Eng $2013 ; 29(12): 1338-60$.

[16] Nigmatullin RR. Fractional integral and its physical interpretation. Teoreticheskaya i Matematicheskaya Fizika 1992;90(3):354-68.

[17] Nigmatullin RR, Le Mehaute A. Is there geometrical/physical meaning of the fractional integral with complex exponent? J Non-Cryst Solids 2005;351:2888-99.

[18] Carpinteri A, Cornetti P. A fractional calculus approach to the description of stress and strain localization in fractal media. Chaos Solitons Fract 2002;13:85-94.

[19] Carpinteri A, Chiaia B, Cornetti P. On the mechanics of quasi-brittle materials with a fractal microstructure. Eng Fract Mech 2003;70:2321-49. 
[20] Kolwankar KM, Gangal AD. Local fractional Fokker-Planck equation. Phys Rev Lett 1998;80:214-7.

[21] Ren F-Y, Yu Z-G, Su F. Fractional integral associated to the self-similar set or the generalized self-similar set and its physical interpretation. Phys Lett A 1996;219(1-2):59-68.

[22] Metzler R, Klafter J. The random walk's guide to anomalous diffusion: a fractional dynamics approach. Phys Rep 2000;339:1-77.

[23] Metzler R, Klafter J, Sokolov M. Anomalous transport in external fields: continuous time random walks and fractional diffusion equations extended. Phys Rev E 1998;58:1621.

[24] Mandelbrot BB. The fractal geometry of nature. San Francisco: Freeman; 1982.

[25] Carpinteri A. Fractal nature of material microstructure and size effects on apparent mechanical properties. Mech Mater 1994;18:89-101.

[26] Butera S, Di Paola M. A physical approach to the connection between fractal geometry and fractional calculus. In: IEEE transactions of the 2014 international conference on fractional differentiation and its application; June 2014.

[27] Alaimo G, Zingales M. A physical description of fractional-order fourier diffusion. In: IEEE transactions of the 2014 international conference on fractional differentiation and its application; June 2014.

[28] Sapora A, Cornetti P, Carpinteri A. Wave propagation in non-local elastic continua modelled by a fractional calculus approach. Commun Nonlinear Sci Numer Simul 2013;18:63-74.

[29] Atanackovic T, Grillo A, Wittum G, Zorica D. Fractional Jeffreys-type diffusion equation. In: Fourth IFAC workshop fractional differentiation and its applications (FDA10); October 2010.

[30] Nigmatullin RR, Baleanu D. The derivation of the generalized functional equations describing self-similar processes. Fract Calc Appl Anal 2012;15(4):718-40.

[31] Falconer K. Mathematical foundations and applications fractal geometry. New York: John Wiley and Sons; 1993.

[32] Samko SG, Kilbas AA, Marichev OI. Fractional integrals and derivatives: theory and applications. Taylor \& Francis; 1987. 